\title{
Asteroseismic stellar activity relations
}

\author{
A. Bonanno ${ }^{1}$, E. Corsaro ${ }^{2,1}$, and C. Karoff ${ }^{3,4}$ \\ 1 INAF - Osservatorio Astrofisico di Catania, via S. Sofia, 78, 95123 Catania, Italy \\ e-mail: alfio.bonanno@inaf.it \\ 2 Instituut voor Sterrenkunde, KU Leuven, Celestijnenlaan 200D, 3001 Leuven, Belgium \\ 3 Stellar Astrophysics Centre, Department of Physics and Astronomy, Aarhus University, Ny Munkegade 120, 8000 Aarhus C, \\ Denmark \\ 4 Department of Geoscience, Aarhus University, Høegh-Guldbergs Gade 2, 8000 Aarhus C, Denmark
}

Received 18 July 2014 / Accepted 12 September 2014

\begin{abstract}
Context. In asteroseismology an important diagnostic of the evolutionary status of a star is the small frequency separation which is sensitive to the gradient of the mean molecular weight in the stellar interior. It is thus interesting to discuss the classical ageactivity relations in terms of this quantity. Moreover, as the photospheric magnetic field tends to suppress the amplitudes of acoustic oscillations, it is important to quantify the importance of this effect by considering various activity indicators.

Aims. We propose a new class of age-activity relations that connects the Mt. Wilson $S$ index and the average scatter in the light curve with the small frequency separation and the amplitude of the p-mode oscillations.

Methods. We used a Bayesian inference to compute the posterior probability of various empirical laws for a sample of 19 solar-like active stars observed by the Kepler telescope.

Results. We demonstrate the presence of a clear correlation between the Mt. Wilson $S$ index and the relative age of the stars as indicated by the small frequency separation, as well as an anti-correlation between the $S$ index and the oscillation amplitudes. We argue that the average activity level of the stars shows a stronger correlation with the small frequency separation than with the absolute age that is often considered in the literature.

Conclusions. The phenomenological laws discovered in this paper have the potential to become new important diagnostics to link stellar evolution theory with the dynamics of global magnetic fields. In particular we argue that the relation between the Mt. Wilson $S$ index and the oscillation amplitudes is in good agreement with the findings of direct numerical simulations of magneto-convection.
\end{abstract}

Key words. stars: activity - stars: oscillations - stars: chromospheres - methods: statistical

\section{Introduction}

It is well known that in an active star the average activity level decreases as the star evolves along the main sequence because it reduces its rotation rate (Skumanich 1972). The observed correlation between X-ray luminosity and rotation rate in late-type stars (Pallavicini et al. 1981) can in fact be explained in terms of a dynamo action, although the precise nature of the underlying dynamo model is still a subject of debate (Wright et al. 2011). Most of the attempts to interpret the correlation between the rotation rate, age, and the amplitude of the activity cycles in a unifying picture have been hampered by serious theoretical difficulties (Noyes et al. 1984; Saar \& Brandenburg 1999; Böhm-Vitense 2007).

The core of the problem is that during various stages of the star's lifetime the type of dynamo responsible for sustaining the star's global magnetic field can change drastically. Young, fastrotating stars approaching the zero-age main-sequence are characterized by a strong dynamo action, a possible nonlinear combination of $\alpha \Omega$ or $\alpha^{2}$ mechanisms, producing irregular activity cycles with large photospheric flux contrast (Bonanno 2013a,b). On the contrary, main-sequence stars with extended convective zones can display regular activity cycles because of the presence of a tachocline, the most likely location for the $\alpha$-effect according to Parker (1993).

The high-quality photometric data from the Kepler space telescope (Borucki et al. 2010) has opened new possibilities in the investigation of these issues. In Chaplin et al. (2011) it was argued that the steep fall in the observed fraction of detected stars with solar-like oscillations has a stellar explanation. Although it was not possible to precisely quantify this effect in terms of chromospheric flux, it is conceivable to assume that the presence of activity suppresses the amplitude of oscillations, which hampers the possibility of detecting the stellar oscillations. On the other hand, recent asteroseismic studies (Karoff et al. 2013; Garcia et al. 2014; Mathur et al. 2014) have further clarified the classical relations between stellar age, rotation, and various activity proxies, generally confirming the basic fact that the level of activity is anti-correlated with the star's age. Interestingly enough, the analysis of Reinhold et al. (2013) has instead confirmed that stellar differential rotation is only weakly dependent on the rotational period.

An important insight into the above questions has recently been provided by Schröder et al. (2013), where a careful study of the evolutionary status of the Mt. Wilson project stars (Baliunas et al. 1995) has led the authors to conclude that stellar activity in main-sequence stars should decline with the relative mainsequence age, i.e., with the evolutionary status of the star, rather than with the absolute age (see also Reiners \& Mohanty 2012).

The are two aims to this work. First, we show that there is a direct correlation between the Mt. Wilson $S$ index chromospheric activity indicator (hereafter $S_{\mathrm{MW}}$ ) and the small frequency separation $\delta v_{02}=v_{n, l=0}-v_{n-1, l+2}$. The latter is a 
Table 1. Values for the asteroseismic parameters and the range index, together with $1 \sigma$ error bars, used for the relations presented in Sect. 2 .

\begin{tabular}{lrrcc}
\hline \hline KIC ID & \multicolumn{1}{c}{$\begin{array}{c}\Delta v \\
(\mu \mathrm{Hz})\end{array}$} & \multicolumn{1}{c}{$\begin{array}{c}\delta v_{02} \\
(\mu \mathrm{Hz})\end{array}$} & $\begin{array}{c}A_{\max } \\
(\mathrm{ppm})\end{array}$ & $\begin{array}{c}r_{\mathrm{hr}} \\
(\mathrm{ppt})\end{array}$ \\
\hline 01435467 & $70.43 \pm 1.10$ & $4.924 \pm 1.512$ & $6.768 \pm 0.440$ & $0.471 \pm 0.099$ \\
02837475 & $75.66 \pm 1.49$ & $6.787 \pm 2.661$ & $6.787 \pm 0.310$ & $0.193 \pm 0.111$ \\
03733735 & $91.85 \pm 1.67$ & $9.058 \pm 3.808$ & $5.174 \pm 0.351$ & $0.222 \pm 0.017$ \\
04914923 & $88.54 \pm 1.55$ & $5.082 \pm 0.980$ & $\ldots$ & $\ldots$ \\
06116048 & $100.50 \pm 0.59$ & $6.224 \pm 0.949$ & $5.975 \pm 0.340$ & $0.290 \pm 0.118$ \\
06603624 & $109.90 \pm 0.46$ & $5.387 \pm 0.423$ & $5.666 \pm 0.470$ & $0.358 \pm 0.037$ \\
06933899 & $71.85 \pm 0.48$ & $5.100 \pm 0.546$ & $8.448 \pm 0.503$ & $0.318 \pm 0.018$ \\
07206837 & $78.84 \pm 2.38$ & $6.494 \pm 3.726$ & $7.003 \pm 0.480$ & $0.504 \pm 0.150$ \\
08006161 & $149.19 \pm 0.34$ & $10.140 \pm 0.688$ & $2.906 \pm 0.265$ & $0.650 \pm 0.189$ \\
08379927 & $119.94 \pm 0.63$ & $10.655 \pm 1.208$ & $3.568 \pm 0.165$ & $1.682 \pm 0.577$ \\
08694723 & $75.22 \pm 0.65$ & $6.182 \pm 1.032$ & $7.939 \pm 0.359$ & $0.286 \pm 0.031$ \\
09098294 & $109.27 \pm 1.33$ & $5.903 \pm 1.900$ & $5.997 \pm 0.593$ & $0.586 \pm 0.188$ \\
09139151 & $117.09 \pm 1.87$ & $10.484 \pm 2.631$ & $4.624 \pm 0.448$ & $0.602 \pm 0.091$ \\
09139163 & $81.23 \pm 0.87$ & $6.662 \pm 1.397$ & $5.911 \pm 0.290$ & $0.452 \pm 0.061$ \\
10454113 & $105.10 \pm 1.25$ & $9.171 \pm 1.410$ & $4.285 \pm 0.308$ & $0.557 \pm 0.038$ \\
11244118 & $71.33 \pm 0.53$ & $5.208 \pm 0.489$ & $8.953 \pm 0.650$ & $0.577 \pm 0.341$ \\
11253226 & $76.92 \pm 2.56$ & $4.811 \pm 4.126$ & $6.615 \pm 0.312$ & $0.470 \pm 0.187$ \\
12009504 & $87.96 \pm 0.58$ & $6.454 \pm 0.954$ & $6.923 \pm 0.381$ & $0.319 \pm 0.041$ \\
12258514 & $74.69 \pm 0.54$ & $4.854 \pm 0.876$ & $7.000 \pm 0.303$ & $0.456 \pm 0.225$ \\
\hline
\end{tabular}

measure of the He fraction in the stellar core (Aerts et al. 2010; Bonanno et al. 2002), i.e., a precise indicator of the evolutionary status of the star, thus confirming the idea expressed in Schröder et al. (2013). Second, we also show that there is a clear anticorrelation between $S_{\mathrm{MW}}$ and the amplitude of maximum power of the p-mode oscillation envelope, $A_{\max }$. It is then clear that the mechanism observed to suppress the p-mode amplitudes in the Sun (Chaplin et al. 2000) is also present in other Sun-like stars.

In particular we make use of a Bayesian inference to precisely and accurately quantify the functional relations expressing our empirical laws.

\section{New asteroseismic relations}

Our targets are part of the Sounding Stellar Cycles With Kepler program (Karoff et al. 2009, 2013), which combines highprecision photometric observations from Kepler with ground based observations from the Nordic Optical Telescope (NOT). In particular, we shall focus on a sample of $N_{\text {stars }}=19$ mainsequence solar-like stars described and analyzed by Karoff et al. (2013), for which asteroseismic measurements for the large frequency separation, $\Delta v$, and the small frequency separation, $\delta v_{02}$, are made available as computed from the frequencies measured by Appourchaux et al. (2012), according to the method described in Bedding et al. (2004). In addition, we enrich the set of asteroseismic measurements by including the oscillation amplitudes at maximum power $A_{\max }$, obtained by Huber et al. (2011), which we found available for 18 targets of our sample, and by computing another index, known as $r_{\mathrm{hr}}$ or the range, related to the average scatter in the Kepler light curve and derived according to the method described by Basri et al. (2010) by using long cadence Q6 data (see also Chaplin et al. 2011, for more details). All the data presented here are listed in Table 1.

In the context of this work we have investigated new empirical asteroseismic relations that involve the activity proxies $S_{\mathrm{MW}}$ and $r_{\mathrm{hr}}$ and the asteroseismic parameters $\delta v_{02}$ and $A_{\max }$. Similarly to the analysis done by Corsaro et al. (2013) for the amplitude scaling relations in asteroseismology, we have adopted the following equations in logarithmic form:

$$
\begin{aligned}
& \ln S_{\mathrm{MW}}=a_{1}+b_{1} \ln \delta v_{02}, \\
& \ln r_{\mathrm{hr}}=a_{2}+b_{2} \ln \delta v_{02}, \\
& \ln S_{\mathrm{MW}}=a_{3}+b_{3} \ln A_{\max }, \\
& \ln r_{\mathrm{hr}}=a_{4}+b_{4} \ln A_{\max } .
\end{aligned}
$$

The free parameters $\left(a_{i}, b_{i}\right)$ for $i$ ranging from 1 to 4 , are estimated by means of a Bayesian analysis. For this purpose, we exploited the log-likelihood introduced by Corsaro et al. (2013), assuming that the $S$ indices are log-normally distributed, which we write here as

$\Lambda\left(a_{i}, b_{i}\right)=\Lambda_{0}-\frac{1}{2} \sum_{j=1}^{N_{\text {stars }}}\left[\frac{\Delta_{j}\left(a_{i}, b_{i}\right)}{\widetilde{\sigma}_{j}\left(b_{i}\right)}\right]^{2}$

with

$\Lambda_{0}=-\sum_{j=1}^{N_{\text {stars }}} \ln \sqrt{2 \pi \widetilde{\sigma}_{j}\left(b_{i}\right)}$

and

$\Delta_{j}\left(a_{i}, b_{i}\right)=\ln S_{\mathrm{MW}, j}^{\mathrm{obs}}-\ln S_{\mathrm{MW}, j}^{\mathrm{pred}}\left(a_{i}, b_{i}\right)$,

namely the difference between the measured $S$ index provided by Karoff et al. (2013) and the one predicted by the empirical relations for the $j$ th star. Uniform priors are adopted for all the free parameters. Still following Corsaro et al. (2013), we point out that the relative uncertainties $\widetilde{\sigma}_{j}\left(b_{i}\right)$ for each target are obtained according to the formal expression of the relations investigated, hence yielding

$\widetilde{\sigma}_{j}^{2}\left(b_{1}\right)=\left(\frac{\sigma_{S_{\mathrm{MW}, j}}}{S_{\mathrm{MW}, j}}\right)^{2}+b_{1}^{2}\left(\frac{\sigma_{\delta v_{02, j}}}{\delta v_{02, j}}\right)^{2}$ 
Table 2. Mean values of the free parameters (offsets $a_{i}$ and slopes $b_{i}$ ) indicated by Eqs. (1) to (4) and Eqs. (10) and (11), with corresponding 68.3\% Bayesian credible intervals.

\begin{tabular}{lrr}
\hline \hline Empirical relation & Offset $\left(a_{i}\right)$ & \multicolumn{1}{c}{ Slope $\left(b_{i}\right)$} \\
\hline$S_{\mathrm{MW}}-\delta v_{02}$ & $-2.33_{-0.04}^{+0.06}$ & $0.25_{-0.03}^{+0.02}$ \\
$r_{\mathrm{hr}}-\delta v_{02}$ & $-2.94_{-0.31}^{+0.49}$ & $1.09_{-0.27}^{+0.17}$ \\
$S_{\mathrm{MW}}-A_{\max }$ & $-0.93_{-0.08}^{+0.06}$ & $-0.50_{-0.03}^{+0.04}$ \\
$r_{\mathrm{hr}}-A_{\max }$ & $1.20_{-0.41}^{+0.33}$ & $-1.21_{-0.18}^{+0.22}$ \\
$S_{\mathrm{MW}}-$ Age & $-1.62_{-0.04}^{+0.03}$ & $-0.13_{-0.01}^{+0.02}$ \\
$S_{\mathrm{MW}}-\Delta v$ & $-3.46_{-0.04}^{+0.06}$ & $0.36_{-0.01}^{+0.01}$ \\
\hline
\end{tabular}

and similarly

$\widetilde{\sigma}_{j}^{2}\left(b_{3}\right)=\left(\frac{\sigma_{S_{\mathrm{MW}, j}}}{S_{\mathrm{MW}, j}}\right)^{2}+b_{3}^{2}\left(\frac{\sigma_{A_{\max , j}}}{A_{\max , j}}\right)^{2}$

for Eq. (1) and Eq. (3), respectively, with $\sigma_{S_{\mathrm{MW}}}, \sigma_{\delta v_{02}}$, and $\sigma_{A_{\max }}$ being the standard uncertainties coming from the dataset adopted. This is done in order to take into account the indeterminacy on both coordinates. A perfectly analogous treatment is applied to Eq. (2) and Eq. (4) by simply replacing $S_{\mathrm{MW}}$ with $r_{\mathrm{hr}}$.

To provide additional arguments in support of the relation between stellar activity and relative age of the star, we also consider in our analysis a relation between the $S$ index and the absolute stellar age (hereafter Age for simplicity) and another between the $S$ index and the large frequency separation of $p$ modes $(\Delta v)$ known to be sensitive to the evolution of the star as it probes the mean stellar density (Ulrich 1986). In particular, we exploit the stellar ages derived by Karoff et al. (2013) by means of asteroseismic modeling, and therefore apply a Bayesian inference along the same lines described previously in this section to the empirical laws

$\ln S_{\mathrm{MW}}=a_{5}+b_{5} \ln \mathrm{Age}$

and

$\ln S_{\mathrm{MW}}=a_{6}+b_{6} \ln \Delta v$.

\subsection{Results}

The results of the Bayesian parameter estimation are listed in Table 2, while the fitted relations are shown in Fig. 1 for Eqs. (1) and (2), in Fig. 2 for Eqs. (3) and (4), and in Fig. 3 for Eqs. (10) and (11).

Given the number of stars used in the inference, the coefficients of the relations for the activity index $S_{\text {MW }}$ appear to be quite well constrained (on average around 7\%), ensuring enough confidence on the detected trends, even in the case of $\delta v_{02}$ despite its large error bars (up to 25\%). For the case of $r_{\mathrm{hr}}$ instead, the constraining level is much weaker (up to $34 \%$ ), showing that for the sample analyzed in this work the $S$ index enables us to obtain a more precise and reliable calibration of the asteroseismic activity relations by a factor of $\sim 5$. This result relies on both the much larger relative error bars of the range index measurements as compared to those of the $S$ index (on average $\sim 26 \%$ and $\sim 1 \%$ for $r_{\mathrm{hr}}$ and $S_{\mathrm{MW}}$, respectively) and on the different dispersion of the data points relative to the fit, as one can derive by comparing the weighted standard deviation of the residuals of the fit (on average $\sim 0.30$ and $\sim 0.08$ for $r_{\mathrm{hr}}$ and $S_{\mathrm{MW}}$, respectively).
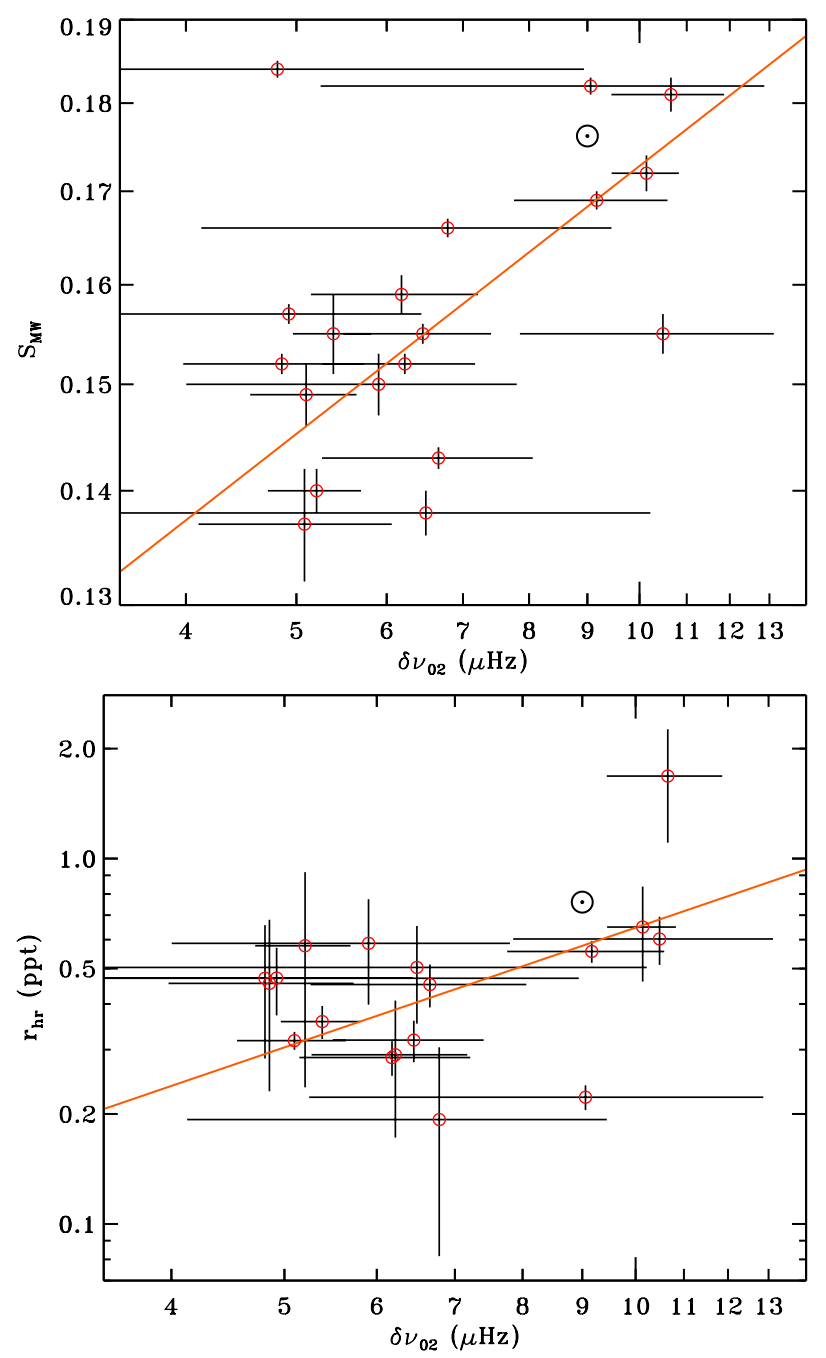

Fig. 1. Log-log scale plot of the $S$ index (top) and range index (bottom) as a function of the small frequency separation $\delta v_{02}$. The orange solid line indicates the resulting Bayesian fits to Eqs. (1) and (2), respectively, while the open symbols are the measured quantities, with corresponding error bars overlaid on both axes. The Sun symbol for the range index corresponds to the value adopted by Basri et al. (2010).

However, apart from the significance of the fits for the two different indices, by looking at the results shown in this work we can clearly see that the stellar activity indicated by both $r_{\mathrm{hr}}$ and $S_{\mathrm{MW}}$ increases towards larger values of $\delta v_{02}$ (with a slope $b_{1} \simeq 0.25$ for $S_{\mathrm{MW}}$ and $b_{3} \simeq 1.09$ for $r_{\mathrm{hr}}$ ). When comparing this result with that obtained through the relation $S_{\mathrm{MW}}$-Age, we find that $S_{\text {MW }}$ correlates with the absolute stellar age with residuals dispersed twice as much as the case $S_{\mathrm{MW}}-\delta v_{02}$, and that the relation $S_{\mathrm{MW}}$-Age also has parameter estimates with relative error bars about two times larger than for $S_{\mathrm{MW}}-\delta v_{02}$, together with a slope $b_{5} \simeq 0.5 b_{1}$, proving that the dependence of stellar activity is weaker with the absolute age of the star. Finally, the relation $S_{\mathrm{MW}}-\Delta v$ gives residuals that are on average about two times more dispersed than for $S_{\mathrm{MW}}-\delta v_{02}$, although for the dataset used the relative error bars on $\Delta v$ are about 20 times smaller than for $\delta v_{02}$. In other words, this shows that the measured activity level of the stars in our sample is significantly more sensitive to their relative main-sequence age than to the absolute age, thus validating the argument exposed by Schröder et al. (2013).

For the case of the p-mode oscillation amplitude $A_{\max }$, we clearly see that for both the $S$ and range indices, higher activity 

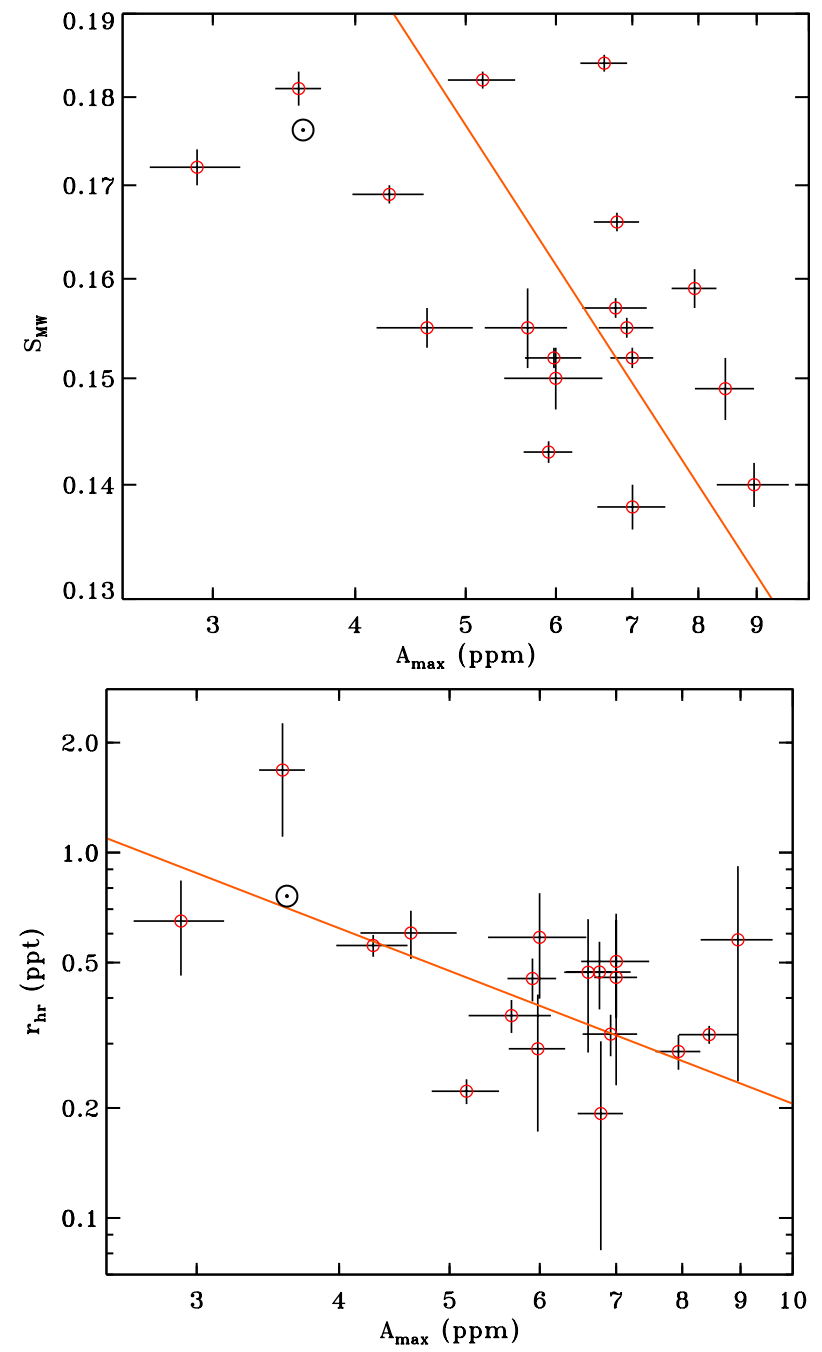

Fig. 2. Same as in Fig. 1, but in the case of the relation relative to $S_{\mathrm{MW}}$ (top) and $r_{\mathrm{hr}}$ (bottom) with $A_{\max }$, given by Eqs. (3) and (4), respectively.

levels correspond to lower oscillation amplitudes, in agreement with the expected suppression of the oscillation signal shown by Chaplin et al. (2011), which we quantify here with a slope $b_{2} \simeq-0.50$ for $S_{\mathrm{MW}}$ and $b_{4} \simeq-1.21$ for $r_{\mathrm{hr}}$.

Finally, we point out that we have tested the relations presented in Sect. 2 with the activity index $\log \left\langle\Delta \mathcal{F}_{\mathrm{Ca}}\right\rangle$ as provided by Karoff et al. (2013), thus confirming the same trends found in the case of the $S$ index.

\subsection{Correlating $S$ and range indices}

In the previous section we have shown that both the $S$ and the range indices correlate similarly to the asteroseismic parameters $\delta v_{02}$ and $A_{\max }$. However, the range index has not yet been proven to correlate directly with the stellar activity of the star. In order to test the reliability of the range index to probe the stellar activity level we also performed the Bayesian inference on the relation

$\ln S_{\mathrm{MW}}=\alpha+\beta \ln r_{\mathrm{hr}}$,

hence estimating the parameters $(\alpha, \beta)$ by adopting once again similar arguments to those already used for Eqs. (1) to (4). The result of the parameter estimation is shown in Fig. 4, where we can observe a direct correlation between the two indices, as already expected from the previous results shown in this work.
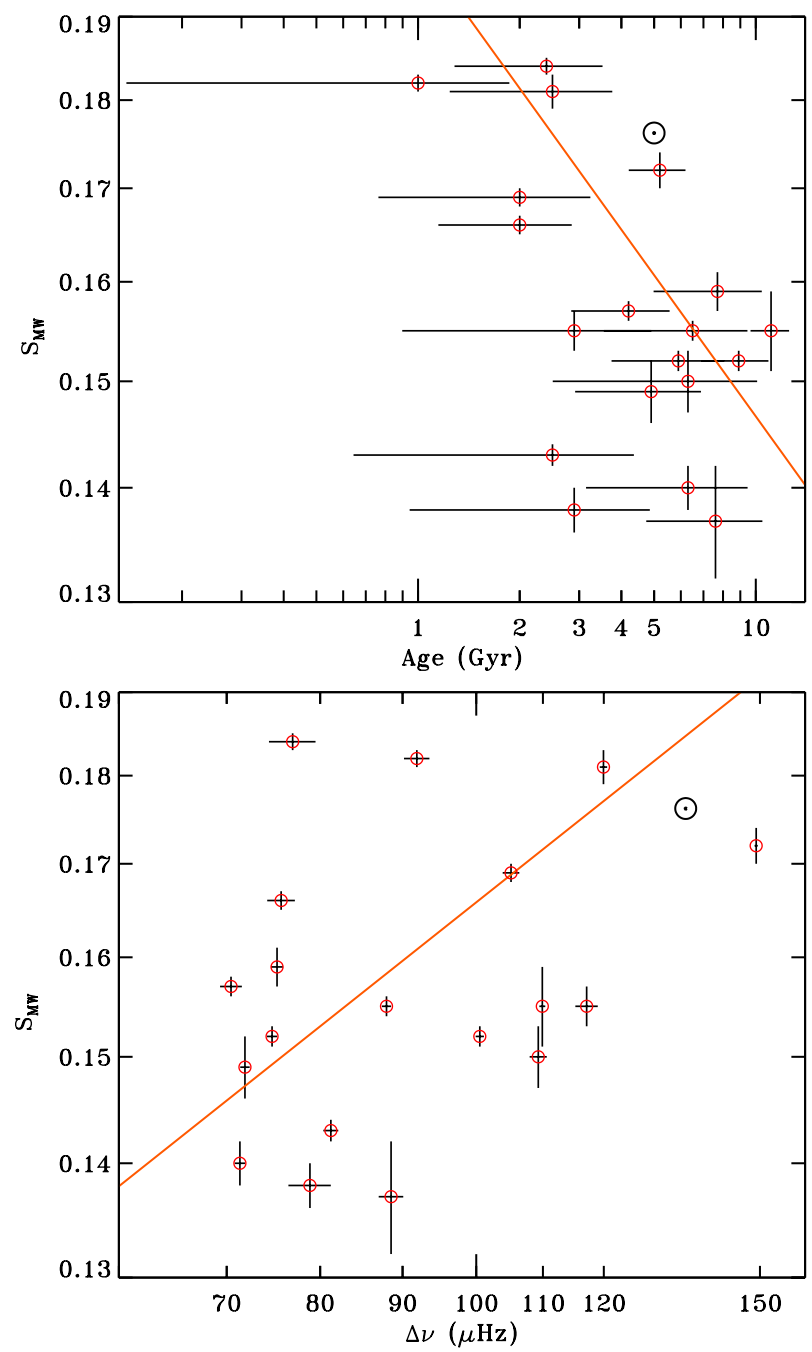

Fig. 3. Same as in Fig. 1, but in this case for the relations relative to $S_{\mathrm{Mw}}$ with Age (top) and $\Delta v$ (bottom) given by Eqs. (10) and (11).

However, the data points appear dispersed, with residuals of about $30 \%$, which together with the mean offset $\alpha=-1.09_{-0.18}^{+0.10}$ and slope $\beta=0.75_{-0.18}^{+0.10}$ (very steep variation in the $S$ index) of Eq. (12) show that for the given sample of stars, $S_{\mathrm{MW}}$ and $r_{\mathrm{hr}}$ are not strongly correlated.

\section{Discussion and conclusions}

Can we explain the phenomenological law given by Eq. (3) in terms of basic physical processes occurring at the chromospheric and photospheric level of the star? In the case of the Sun it has been shown that the $S$ index is roughly proportional to the local field strength $B$ (Skumanich et al. 1975). It is thus obvious to assume that a relation of the type $S_{\mathrm{MW}} \propto B$ holds to a good approximation for the solar-type stars considered in this study. On the other hand, direct numerical simulation of magneto-convection (Jacoutot et al. 2008) have proven that the power of acoustic oscillations is inversely proportional to the local field strength (see in particular their Fig. 4). This is in agreement with our findings for which $A_{\max } \propto 1 / S_{\mathrm{MW}}^{2} \propto 1 / B^{2}$. We think that this result is interesting because it opens up the possibility of obtaining important information on the local structure of the chromospheric field by means of asteroseismology. 
A. Bonanno et al.: Asteroseismic stellar activity relations

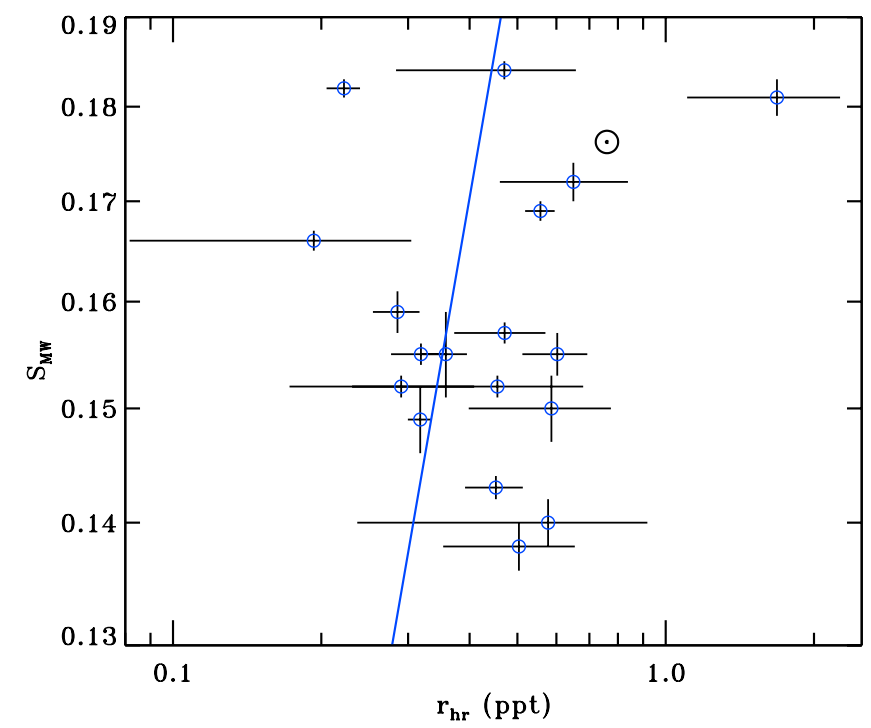

Fig. 4. Same as in Fig. 1, but in the case of the relation $S_{\mathrm{MW}}-r_{\mathrm{hr}}$, given by Eq. (12).

It is also important to stress that, while most of the studies on age-activity-rotation present in the literature have focussed on clusters younger than about 500 Myrs, in this work we used asteroseismology to address the age-activity relation in mainsequence and subgiant stars demonstrating the occurrence of a clear correlation between the small frequency separation of p-mode oscillations $\delta v_{02}$ and the activity index $S_{\text {MW }}$ in a sample of 19 solar-like stars. Our findings suggest that a more suitable candidate with which to study the age-activity relation is $\delta v_{02}$ instead of the absolute age derived through asteroseismic modeling, which, unfortunately, is the least constrained quantity in asteroseismic studies (Metcalfe et al. 2010). We have also shown that the suppression of the p-modes energy in the pulsational spectrum due to the presence of a photospheric field is efficient, and leads to a rapid decrease in the p-mode amplitudes in very active stars. Although a clear limitation of our study is the impossibility of extending this analysis to very young, fast rotating stars with strong magnetic fields, we argue that the idea proposed in Schröder et al. (2013) is essentially correct.

Our testing of a possible connection between the two indices $S_{\mathrm{MW}}$ and $r_{\mathrm{hr}}$ has shown that the very steep variation observed, together with the large dispersion of the data points, do not provide any evidence for a strong correlation. We therefore conclude that for our sample of stars the two indices are not equally sensitive to their activity level, $S_{\mathrm{MW}}$ being the more sensitive one, although this result would require a larger number of targets with $S$ index measurements available in order to be confirmed and extended to a wider range of stellar properties. Moreover, the use of the $S_{\mathrm{MW}}$ index instead of $r_{\mathrm{hr}}$, has a direct physical interpretation as explained above.

It is important to further investigate our new phenomenological relations using larger stellar samples and we hope to address this question in the near future.

Acknowledgements. The research leading to these results has received funding from the European Research Council under the European Community's Seventh Framework Programme (FP7/2007-2013) ERC grant agreement $n^{\circ} 227224$ (PROSPERITY), from the Fund for Scientific Research of Flanders (G.0728.11), and from the Belgian federal science policy office (C90291 Gaia-DPAC). C.K. acknowledges support from the Villum foundation. Funding for the Stellar Astrophysics Centre is provided by The Danish National Research Foundation (grant agreement No.: DNRF106).

\section{References}

Aerts, C., Christensen-Dalsgaard, J., \& Kurtz, D. W. 2010, Asteroseismology (Springer)

Appourchaux, T., Chaplin, W. J., García, R. A., et al. 2012, A\&A, 543, A54

Baliunas, S. L., Donahue, R. A., Soon, W. H., et al. 1995, ApJ, 438, 269

Basri, G., Walkowicz, L. M., Batalha, N., et al. 2010, ApJ, 713, L155

Bedding, T. R., Kjeldsen, H., Butler, R. P., et al. 2004, ApJ, 614, 380

Böhm-Vitense, E. 2007, ApJ, 657, 486

Bonanno, A. 2013a, Sol. Phys., 287, 185

Bonanno, A. 2013b, Mem. Soc. Astron. It., 84, 315

Bonanno, A., Schlattl, H., \& Paternò, L. 2002, A\&A, 390, 1115

Borucki, W. J., Koch, D., Basri, G., et al. 2010, Science, 327, 977

Chaplin, W. J., Elsworth, Y., Isaak, G. R., Miller, B. A., \& New, R. 2000, MNRAS, 313, 32

Chaplin, W. J., Bedding, T. R., Bonanno, A., et al. 2011, ApJ, 732, L5

Corsaro, E., Fröhlich, H.-E., Bonanno, A., et al. 2013, MNRAS, 430, 2313

Garcia, R. A., Ceillier, T., Salabert, D., et al. 2014, A\&A, in press DOI: 10.1051/0004-6361/201423888

Huber, D., Bedding, T. R., Stello, D., et al. 2011, ApJ, 743, 143

Jacoutot, L., Kosovichev, A. G., Wray, A., \& Mansour, N. N. 2008, ApJ, 684, L51

Karoff, C., Metcalfe, T. S., Chaplin, W. J., et al. 2009, MNRAS, 399, 914

Karoff, C., Metcalfe, T. S., Chaplin, W. J., et al. 2013, MNRAS, 433, 3227

Mathur, S., García, R. A., Ballot, J., et al. 2014, A\&A, 562, A124

Metcalfe, T. S., Monteiro, M. J. P. F. G., Thompson, M. J., et al. 2010, ApJ, 723, 1583

Noyes, R. W., Weiss, N. O., \& Vaughan, A. H. 1984, ApJ, 287, 769

Pallavicini, R., Golub, L., Rosner, R., et al. 1981, ApJ, 248, 279

Parker, E. N. 1993, ApJ, 408, 707

Reiners, A., \& Mohanty, S. 2012, ApJ, 746, 43

Reinhold, T., Reiners, A., \& Basri, G. 2013, A\&A, 560, A4

Saar, S. H., \& Brandenburg, A. 1999, ApJ, 524, 295

Schröder, K.-P., Mittag, M., Hempelmann, A., González-Pérez, J. N., \& Schmitt, J. H. M. M. 2013, A\&A, 554, A50

Skumanich, A. 1972, ApJ, 171, 565

Skumanich, A., Smythe, C., \& Frazier, E. N. 1975, ApJ, 200, 747

Ulrich, R. K. 1986, ApJ, 306, L37

Wright, N. J., Drake, J. J., Mamajek, E. E., \& Henry, G. W. 2011, ApJ, 743, 48 